\title{
Effects of internal radiation on heat flow and facet formation in Bridgman growth of YAG crystals
}

\author{
C.W. Lan *, C.Y. Tu, Y.F. Lee \\ Department of Chemical Engineering, National Taiwan University, Taipei 10617, Taiwan, ROC
}

Received 14 February 2002; accepted 9 September 2002

\begin{abstract}
Internal radiation, in the crystal and the melt, is investigated using the $P_{1}$-approximation for the Bridgman growth of YAG crystals. Its effects on the interface shape and facet formation are illustrated through three-dimensional simulation. The $P_{1}$-approximation is first validated by a one-dimensional solution. Further comparison for axisymmetric cases with the rigorous calculations by Brandon and Derby [J. Crystal Growth 121 (1992) 473] is performed for opaque melt, and reasonable agreement is obtained for optical distance being $<1 \mathrm{~cm}$. The no-slip Rosseland model also gives a reasonable prediction in the interface shape; however, interface position and facet size are over predicted due to the poor approximation in the thermal gradients at the interface. Furthermore, melt transparency introduces radiation heating from the hot zone to the interface. Accordingly, the interface concavity is reduced with the increasing optical distance of the melt.
\end{abstract}

(c) 2003 Elsevier Science Ltd. All rights reserved.

Keywords: Internal radiation; Facet formation; Convection; 3D simulation; Bridgman growth

\section{Introduction}

Radiation in a participating medium, or the so-called internal radiation, is important in the crystal growth of semitransparent crystals like oxides. Both experimental $[1,2]$ and theoretical [3-6] works have shown that the internal radiation significantly affects the interface shape, which is crucial to crystal quality. Morphological instability can be induced as well [7]. For the growth of oxide crystals, the induced facet formation at the growing interface is particularly important. For example, the growth of YAG or GGG in the $\langle 111\rangle$ direction, faceting appears at $\{211\}$ crystallographic planes [8]. Faceting can affect the crystal quality in several ways. The most common ones are the stress concentration at the singular edges and the severe segregation due to different segregation coefficients at the singular facets. The dark core in the growth of $\mathrm{Bi}_{12} \mathrm{SiO}_{20}$ is also an example of the ion segregation due to faceting [9]. There-

\footnotetext{
${ }^{*}$ Corresponding author. Fax: +886-2-23633917.

E-mail address: cwlan@ntu.edu.tw (C.W. Lan).
}

fore, the control of facet formation is an important task in the improvement of crystal quality, and computer simulation could be very useful in tuning growth conditions. However, most of the facets observed in experiments, such as $\{211\}$ or $\{110\}$ facets, are not axisymmetric. Therefore, a three-dimensional (3D) model is necessary for taking these facets into account, and this has not yet been carried out until recently. By using a finite volume method (FVM), Lan and Tu [10] presented, for the first time, the 3D simulation of facet formation in the Bridgman growth of YAG crystals. The interface shape and the facets, as well as the coupled flow and dopant distribution, are predicted for various growth conditions. However, their internal radiation was treated by the simple no-slip Rosseland diffusion approach, which is valid only for optically thick materials. For most of oxides, except with high doping, the crystal (maybe the melt as well) is usually optically transparent, i.e., having a large optical distance. In such a case, a more rigorous model is necessary for a better simulation.

An accurate treatment of the internal radiation is not a trivial task, even for a $2 \mathrm{D}$ geometry. Brandon and 


\begin{tabular}{|c|c|c|c|}
\hline \multicolumn{4}{|c|}{ Nomenclature } \\
\hline$a$ & optical absorption coefficient & $R$ & radius of curvature at the interface \\
\hline$\tilde{a}$ & normalized optical absorption coefficient & $R_{\mathrm{a}}$ & ampoule radius \\
\hline$a_{\mathrm{R}}$ & Rosseland optical absorption coefficient & $\operatorname{Rad}$ & radiation number, $\mathrm{Rad}=\sigma \varepsilon_{\mathrm{a}} T_{\mathrm{m}}^{3} D_{\mathrm{c}} / k_{\mathrm{m}}$ \\
\hline$A$ & facet plane constant & $R a_{\mathrm{T}}$ & thermal Rayleigh number, $g \beta_{\mathrm{T}} D_{\mathrm{c}}^{3} T_{\mathrm{m}} / v_{\mathrm{m}} \alpha_{\mathrm{m}}$ \\
\hline$b$ & facet size & $R_{\mathrm{c}}$ & crystal radius \\
\hline$B$ & facet plane constant & St & Stefan number, $\Delta H / C p_{\mathrm{m}} T_{\mathrm{m}}$ \\
\hline$B i$ & Biot number, $B i=h D_{\mathrm{c}} / k_{\mathrm{m}}$ & $T$ & temperature \\
\hline$c$ & kinetic exponent & $T_{1}$ & temperature at wall 1 \\
\hline C & facet plane constant & $T_{2}$ & temperature at wall 2 \\
\hline$C_{p}$ & specific heat & $T_{\mathrm{a}}$ & ambient temperature distribution \\
\hline$d$ & adiabatic zone length & $T_{\mathrm{C}}$ & cold zone temperature \\
\hline$D$ & facet plane constant & $T_{\mathrm{H}}$ & hot zone temperature \\
\hline$D_{\mathrm{c}}$ & crystal diameter & $T_{\mathrm{m}}$ & melting point \\
\hline$e_{\mathrm{g}}$ & unit vector in the gravity direction & $\Delta T_{\text {kin }}$ & kinetic supercooling \\
\hline $\boldsymbol{e}_{x}$ & unit vector in $x$-direction & $u$ & $x$-component of velocity \\
\hline $\boldsymbol{e}_{y}$ & unit vector in $y$-direction & $U_{\mathrm{a}}$ & ampoule pulling speed \\
\hline $\boldsymbol{e}_{z}$ & unit vector in $z$-direction & $U_{\mathrm{n}}$ & normal speed of the interface \\
\hline$f$ & facet plane function & $v$ & $y$-component of velocity \\
\hline$g$ & gravitational acceleration & $v$ & velocity vector \\
\hline$G_{n}$ & local thermal gradient at the interface & $w$ & $z$-component of velocity \\
\hline$G_{\mathrm{T}}$ & $\begin{array}{l}\text { thermal gradient of adiabatic zone, } G_{\mathrm{T}}= \\
\left(T_{\mathrm{H}}-T_{\mathrm{C}}\right) / d\end{array}$ & \multicolumn{2}{|c|}{ Greek symbols } \\
\hline$h$ & heat transfer coefficient & $\alpha_{\mathrm{m}}$ & thermal diffusivity of melt \\
\hline$h_{\mathrm{m}}$ & interface height & $\beta$ & kinetic coefficient \\
\hline$\Delta H$ & heat of fusion & $\beta_{\mathrm{T}}$ & thermal expansion coefficient \\
\hline$J$ & dimensionless irradiance & $\varepsilon$ & surface emissivity \\
\hline$k$ & thermal conductivity & $\gamma$ & density ratio, $\rho / \rho_{\mathrm{m}}$ \\
\hline$k^{\text {cond }}$ & molecular thermal conductivity & $\begin{array}{c}\kappa \\
\theta\end{array}$ & thermal conductivity ratio, $\kappa=k / k_{\mathrm{m}}$ \\
\hline$L$ & sample length & $\sigma$ & Stefan Boltzmann constant \\
\hline$n$ & refractive index & 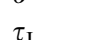 & dimensionless optical distance, $\tau_{\mathrm{L}}=a L$ \\
\hline$\tilde{n}$ & normalized refractive index & $v_{\mathrm{m}}$ & kinematic viscosity \\
\hline$N$ & conduction to radiation ratio, $N=$ & \multicolumn{2}{|c|}{ Subscripts } \\
\hline & $k_{\mathrm{c}} a_{\mathrm{c}} / 4 \sigma T_{1}^{3}$ & $\mathrm{a}$ & ampoule \\
\hline$N_{\mathrm{R}}$ & radiation to conduction ratio, $N_{\mathrm{R}}=$ & $\mathrm{c}$ & crystal \\
\hline & $4 n_{\mathrm{m}}^{2} \sigma T_{\mathrm{m}}^{3} D_{\mathrm{c}} / k_{\mathrm{m}}$ & $\mathrm{m}$ & melt \\
\hline$P$ & Pressure & $x$ & $x$-direction in the Cartesian coordinate \\
\hline $\mathrm{Pe}$ & Peclet number, $P e=\rho C_{p} U_{\mathrm{amp}} D_{\mathrm{c}} / k_{\mathrm{m}}$ & $y$ & $y$-direction in the Cartesian coordinate \\
\hline $\operatorname{Pr}$ & Prandtl number, $v_{\mathrm{m}} / \alpha_{\mathrm{m}}$ & $z$ & $z$-direction in the Cartesian coordinate \\
\hline
\end{tabular}

Derby [3] conducted the first modeling of internal radiation in Bridgman growth of oxide crystals using a rigorous finite element method. Since their solution involved a finite-element solution of differential-integral equations, the calculation was extremely tedious and timeconsuming. On the other hand, if the material does not participate the radiation (zero absorption coefficient), the face-to-face radiation can be treated by view factors [4], and this can save computational cost dramatically. However, this situation is rare in practice. In fact, both approaches are time-consuming for 3D simulation.
To compromise the accuracy and computational load, a tradeoff is to use the $P_{N}$-approximation, which expands the radiation intensity by an orthogonal series of spherical harmonics [11]. Its simplest form, i.e., the $P_{1}$ approximation, is used most because it requires only the solution of a diffusion-like equation. Matsushima and Viskanta [12] used the $P_{1}$-approximation to simulate a simple 2D Bridgman growth of semiconductor crystals including the spectral dependence. They also pointed out that the difference between spectral-band and gray models is small. The effect of internal radiation in the 
melt for a Czochralski growth of lithium niobate crystals was studied recently by Tsukada et al. [5] and Kobayashi et al. [6]. They showed that the melt transparency significantly affects the interface morphology and the critical rotation rate for interface inversion. Although the $P_{1}$ approximation has been widely used in crystal growth simulation, its validity has not yet been examined. In fact, even for the simplest Rosseland diffusion model, the agreement with the most rigorous model is only qualitative $[13,14]$.

Therefore, in this paper we adopt the $P_{1}$-approximation in a $3 \mathrm{D}$ Bridgman model for better simulation of the internal radiation considering faceting. The effects of internal radiation on the heat flow, interface shape, and facet formation are investigated. For comparison purposes, a benchmark comparison with a simple 1D model is performed first. Further comparison is then carried out for the axisymmetric Bridgman crystal growth simulated by Brandon and Derby [3]. From there, the validity of the $P_{1}$ model for a wide range of optical distance can be examined. After the confidence is built, facet formation is considered and its asymmetric effect on the heat flow is discussed. The solution predicted by the no-slip Rosseland diffusion model is also presented for comparison. Finally, the effect of internal radiation in the melt is discussed as well. In the next section, the model and its numerical simulation are briefly described. Section 3 is devoted to the Results and discussion, followed by Conclusions in Section 4.

\section{Model description and numerical solution}

A generic Bridgman crystal growth configuration in shown Fig. 1a. Due to the asymmetric faceting at the interface, the problem is $3 \mathrm{D}$ and thus described by a Cartesian coordinate $(x, y, z)$. The ambient thermal distribution $T_{\mathrm{a}}(x, y, z)$ is assumed to be known. For comparison purposes, the typical three-zone heating profile [3] is used. To focus on the internal radiation and facet formation, we have adopted a pseudo-steady state approximation here. The ampoule pulling speed $U_{\mathrm{a}}$ is assumed to be equal to the axial growth rate.

The flow and temperature fields, as well as the melt/ crystal interface, are also represented in the Cartesian coordinate $(x, y, z)$. The dimensionless variables are defined by scaling length with the crystal diameter $D_{\mathrm{c}}$, velocity with $\alpha_{\mathrm{m}} / D_{\mathrm{c}}$, and pressure with $\rho_{\mathrm{m}} \alpha_{\mathrm{m}}^{2} / D_{\mathrm{c}}^{2}$, where $\rho_{\mathrm{m}}$ is the melt density and $\alpha_{\mathrm{m}}$, the thermal diffusivity. The dimensionless temperature is scaled by the equilibrium melting point $T_{\mathrm{m}}$ (for a rough interface). For the convenience of discussion, the variables used in the equations are all dimensionless unless otherwise stated. The pseudo-steady governing equations describing convection in the melt $(m)$ are as follows:

$\nabla \cdot \boldsymbol{v}=0$

$\boldsymbol{v} \cdot \nabla \boldsymbol{v}=-\nabla P+\operatorname{Pr} \nabla^{2} \boldsymbol{v}-\operatorname{Pr} R a_{\mathrm{T}}(T-1) \boldsymbol{e}_{\mathrm{g}}$,

where $\boldsymbol{v}, P$, and $T$ are the dimensionless velocity, pressure, and temperature, respectively. $\operatorname{Pr}$ is the Prandtl

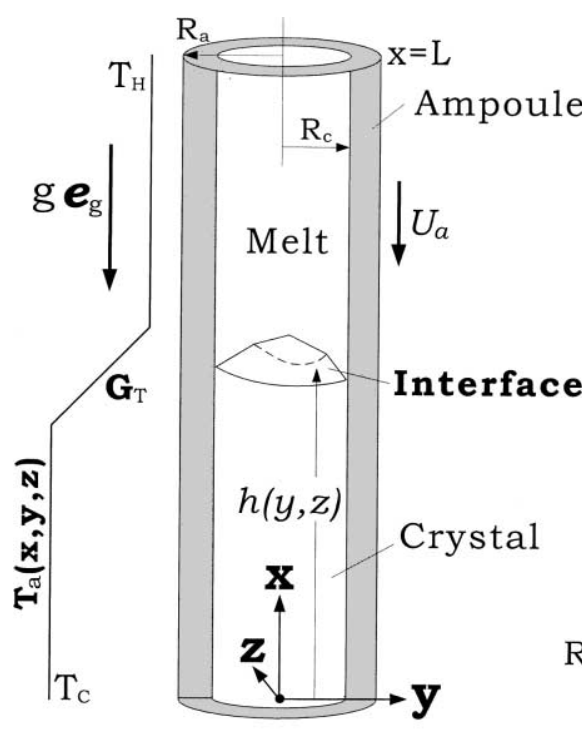

(a)
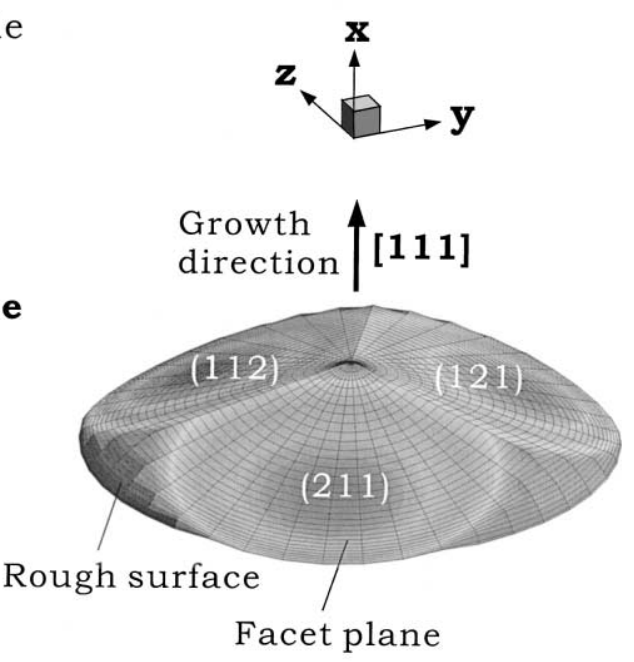

(b)

Fig. 1. (a) Schematic sketch of a Bridgman growth YAG crystal; (b) a sample mesh at the interface with $\{211\}$ facets; the growth direction is in [1 111$]$. 
number $\left(P r \equiv v_{\mathrm{m}} / \alpha_{\mathrm{m}}\right) ; v_{\mathrm{m}}$, the kinematic viscosity. In Eq. (2), the thermal Rayleigh number $R a_{\mathrm{T}}$ in the source term is defined as

$R a_{\mathrm{T}} \equiv \frac{g \beta_{\mathrm{T}} T_{\mathrm{m}} D_{\mathrm{c}}^{3}}{\alpha_{\mathrm{m}} v_{\mathrm{m}}}$,

where $g$ is the gravitational acceleration and $\beta_{\mathrm{T}}$, the thermal expansion coefficient. Furthermore, the gravity unit vector $\boldsymbol{e}_{\mathrm{g}}=-\boldsymbol{e}_{x}$ is pointing downward, as shown in Fig. 1a.

The dimensionless equations for heat transfer in the melt and the crystal are described through the $P_{1}$ approximation as follows:

\section{Melt}

$v \cdot \nabla T=\nabla \cdot \kappa_{\mathrm{m}}(T) \nabla T+\tilde{a}_{\mathrm{m}} N_{\mathrm{R}}\left(J_{\mathrm{m}}-T^{4}\right)$,

Crystal

$P e_{\mathrm{c}} \boldsymbol{e}_{x} \cdot \nabla T=\nabla \cdot \kappa_{\mathrm{c}}(T) \nabla T+\tilde{a}_{\mathrm{c}} N_{\mathrm{R}}\left(J_{\mathrm{c}}-\tilde{n}_{\mathrm{c}}^{2} T^{4}\right)$.

where $\tilde{a}_{i} \equiv a_{i} D_{\mathrm{c}}$ is the dimensionless optical absorption coefficient; $\tilde{n}_{i} \equiv n_{i} / n_{\mathrm{m}}$, the normalized refractive index of phase $i ; J_{i}$, the dimensionless irradiance being scaled by $4 n_{\mathrm{m}} \sigma T_{\mathrm{m}}^{4}$, where $n_{\mathrm{m}}$ and $\sigma$ are the refractive index of the melt and the Stefan Boltzmann constant, respectively. The radiation to conduction ratio $N_{\mathrm{R}} \equiv 4 n_{\mathrm{m}}^{2} \sigma T_{\mathrm{m}}^{3} D_{\mathrm{c}} / k_{\mathrm{m}}$ is an important dimensionless number for the internal radiation. Also, $\kappa_{i}(T) \equiv k_{i}(T) / k_{\mathrm{m}}$ is the dimensionless thermal conductivity of the melt (m) or crystal (c), and $P e_{i} \equiv \rho_{i} C_{p_{i}} U_{\mathrm{a}} D_{\mathrm{c}} / k_{\mathrm{m}}$ is the Peclet number of crystal or ampoule (a); $k_{\mathrm{m}}$, the thermal conductivity of the melt at $T_{\mathrm{m}}$. Also, $\rho_{i}, C_{p_{i}}$, and $k_{i}$ are the density, specific heat, and thermal conductivity of the phase $i(i=\mathrm{c}, \mathrm{m}$, or a), respectively.

The radiative heat transfer is described by the $P_{1}$ approximation for the melt (m) and the crystal (c) is as follows:

$\nabla \cdot \frac{1}{3 \tilde{a}_{i}} \nabla J_{i}-\tilde{a}_{i} N_{\mathrm{R}}\left(J_{i}-\tilde{n}_{i}^{2} T^{4}\right)=0 \quad(i=\mathrm{m}, \mathrm{c})$.

The ampoule is assumed to be opaque, which is reasonable for refractory metals like molybdenum here and the heat transfer is dictated by a typical convective/ conduction heat transfer:

$P e_{\mathrm{a}} \boldsymbol{e}_{x} \cdot \nabla T=\nabla \cdot \kappa_{\mathrm{a}}(T) \nabla T$.

As shown in Eqs. (3) and (4), the $P_{1}$ approximation introduces a source term to consider the internal heating or cooling due to radiation. On the other hand, the Rosseland diffusion approximation is much simpler, without solving any additional differential equations, by treating the thermal conductivity (in the dimensional form) as:

$k_{i}=k_{i}^{\mathrm{cond}}+\frac{16 n_{i}^{2} \sigma T^{3}}{3 a_{\mathrm{R}}} \quad(i=\mathrm{m}, \mathrm{c})$ where $k_{i}^{\text {cond }}$ is the contribution due to conduction and the second term is due to radiation; $a_{\mathrm{R}}$, the Rosseland mean absorption coefficient. Apparently, the internal radiation enhances the heat transfer (heating or cooling effect). Taking the crystal $(i=\mathrm{c})$ as an example, due to the increase of crystal conductivity, the interface convexity increases with the decreasing absorption coefficient $a_{\mathrm{R}}$ [10]. Because the Rosseland approximation is not accurate near the boundary and it is valid only for optically thick materials, we do not anticipate a satisfactory solution from there. Furthermore, for a consistent formation, a jump (slip) boundary condition, such as the Deissler's condition [11], is needed. However, in the recent studies by Vizman et al. $[13,14]$ and Lan and $\mathrm{Tu}$ [10], no special treatment, i.e., the no-slip model, was considered. In this report, we also adopt the no-slip Rosseland diffusion model for comparison. In fact, the use of the slip boundary condition complicates the treatment of the Stefan condition because two temperatures at the interface are necessary for calculation.

The thermal boundary conditions at the melt/solid interfaces are set by the total heat flux balances. For example, at the growth front:

$$
\begin{aligned}
\boldsymbol{n} \cdot & \left.\nabla T\right|_{\mathrm{m}}+\left.\frac{N_{\mathrm{R}}}{3 \tilde{a}_{\mathrm{m}}} \boldsymbol{n} \cdot \nabla J_{\mathrm{m}}\right|_{\mathrm{m}}-\left.\boldsymbol{n} \cdot \kappa_{\mathrm{c}} \nabla T\right|_{\mathrm{c}}-\left.\frac{N_{\mathrm{R}}}{3 \tilde{a}_{\mathrm{c}}} \boldsymbol{n} \cdot \nabla J_{\mathrm{c}}\right|_{\mathrm{c}} \\
& -\gamma_{\mathrm{c}} P e_{\mathrm{m}} S t\left(\boldsymbol{e}_{x} \cdot \boldsymbol{n}\right)=0,
\end{aligned}
$$

where $\vec{n}$ is the unit normal vector at the growth interface pointing to the melt. The Stefan number $S t \equiv$ $\Delta H /\left(C_{p_{\mathrm{m}}} T_{\mathrm{m}}\right)$ scales the heat of fusion $(\Delta H)$ released during solidification to the sensible heat in the melt; $\gamma_{\mathrm{c}}=\rho_{\mathrm{c}} / \rho_{\mathrm{m}}$. In most cases, the melt is assumed to be opaque. Because we still allow an emissivity for the melt, the radiation in the crystal side can be modified as the following for an opaque melt:

$\left.\boldsymbol{n} \cdot \nabla T\right|_{\mathrm{m}}-\left.\boldsymbol{n} \cdot \kappa_{\mathrm{c}} \nabla T\right|_{\mathrm{c}}-\left.\frac{N_{\mathrm{R}}}{3 \tilde{a}_{\mathrm{c}}} \boldsymbol{n} \cdot \nabla J_{\mathrm{c}}\right|_{\mathrm{c}}-\gamma_{\mathrm{c}} P e_{\mathrm{m}} S t\left(\boldsymbol{e}_{x} \cdot \boldsymbol{n}\right)=0$,

where

$-\left.\boldsymbol{n} \cdot \nabla J_{\mathrm{c}}\right|_{\mathrm{c}}=\frac{3 \tilde{a}_{\mathrm{c}} \epsilon_{\mathrm{m}}}{4-2 \epsilon_{\mathrm{m}}}\left(J_{\mathrm{c}}-\tilde{n}_{\mathrm{c}}^{2} T^{4}\right)$,

where $\epsilon_{\mathrm{m}}$ is the surface emissivity of the melt.

Because the ampoule is also opaque, the treatment at the material/ampoule is similar. For example, at the crystal/ampoule interface,

$\left.\boldsymbol{n} \cdot \kappa_{\mathrm{a}} \nabla T\right|_{\mathrm{a}}-\left.\boldsymbol{n} \cdot \kappa_{\mathrm{c}} \nabla T\right|_{\mathrm{c}}-\frac{N_{\mathrm{R}}}{3 \tilde{a}_{\mathrm{c}}} \boldsymbol{n} \cdot \nabla J_{\mathrm{c}}=0$.

Again, the treatment of the boundary condition for the irradiance is similar:

$-\left.\boldsymbol{n} \cdot \nabla J_{\mathrm{c}}\right|_{\mathrm{c}}=\frac{3 \tilde{a}_{\mathrm{c}} \epsilon_{\mathrm{a}}}{4-2 \epsilon_{\mathrm{a}}}\left(J_{\mathrm{c}}-\tilde{n}_{\mathrm{c}}^{2} T^{4}\right)$ 
where $\boldsymbol{n}$ is the unit normal vector pointing to the ampoule and $\epsilon_{\mathrm{a}}$, the surface emissivity of the ampoule. The heat exchange between the ampoule and the furnace is by both radiation and convection according to the energy balance along the ampoule surface,

$\left.\boldsymbol{- n} \cdot \kappa_{\mathrm{a}} \nabla T\right|_{\mathrm{a}}=B i\left(T-T_{\mathrm{a}}\right)+\operatorname{Rad}\left(T^{4}-T_{\mathrm{a}}^{4}\right)$,

where $\boldsymbol{n}$ is the unit normal vector on the ampoule surface pointing outwards; $B i \equiv h D_{\mathrm{c}} / k_{\mathrm{m}}$, the Biot number, and $\operatorname{Rad} \equiv \sigma \epsilon_{\mathrm{a}} T_{\mathrm{m}}^{3} D_{\mathrm{c}} / k_{\mathrm{m}}$ the radiation number. For comparison, the effective furnace temperature $T_{\mathrm{a}}$ is the same as the one used by Brandon and Derby [3]. The hot- and cold-zone temperatures are assumed to be constant, while the temperature profile inside the adiabatic zone $(0.5(L-d)<x<0.5(L+d))$ is assumed linear:

$T_{\mathrm{a}}=T_{\mathrm{m}}+G_{\mathrm{T}}(x-0.5 L)$,

where $G_{\mathrm{T}}$ is the thermal gradient in the adiabatic zone, i.e., $G_{\mathrm{T}}=\left(T_{\mathrm{H}}-T_{\mathrm{C}}\right) / d$, where $d$ is the length of the adiabatic zone. Temperature at the top and bottom surfaces is set to be the furnace temperature there. Although this treatment is somewhat different from that used in [3], the end effect is small.

Also, the temperature at the melt/crystal interface for a rough surface is assumed to be at the equilibrium melting temperature of the material; the supercooling at the rough surface is neglected. For a convex interface, this is a reasonable assumption because the supercooling at the singular faces (facets) is much larger. On the contrary, for a concave interface, the 2D screw growth mechanism shows no superheating in the facet. In such a case, the supercooling at the rough surface becomes important [8]. Due to the internal radiation in the crystal, which is usually much larger than that in the melt, the interface appears to be convex in most of the cases. For a convex interface, if a facet exists, the interface is defined by its crystallographic plane and the highest supercooling in the plane needs to be equal to the kinetic supercooling $\Delta T_{\text {kin }}$. Therefore, at the facet the lowest temperature is defined by

$T=T_{\mathrm{m}}-\Delta T_{\text {kin }}$.

The supercooling $\Delta T_{\text {kin }}$ is a kinetic data and it also depends on the mechanisms of facet formation. In general, for a facet plane, it is related to the growth rate. The simplest form may be taken as the following:

$\Delta T_{\mathrm{kin}}=\beta U_{\mathrm{n}}^{c}=\beta\left[\left|U_{\mathrm{a}}\right|\left(\boldsymbol{n} \cdot \boldsymbol{e}_{x}\right)\right]^{c}$,

where $\beta$ is a kinetic coefficient; $U_{n}$, normal growth rate at the interface, and $c$ is a constant depending on the mechanisms. For example, if the facet is formed by a screw dislocation (growth), $c$ is about 0.5. For a given growth rate, $\Delta T_{\text {kin }}$ also depends on the interface shape. However, for a given facet, $\left(\boldsymbol{n} \cdot \boldsymbol{e}_{x}\right)$ is fixed. Therefore, once a steady-state growth rate is achieved, for a given facet, $\Delta T_{\text {kin }}$ can be used as an input parameter (from the kinetic data). Therefore, for the $\{211\}$ facet, the maximum supercooling is known if the growth rate is specified. In fact, our approach for treating the faceting is similar to the one proposed by Brice [1] and Voronkov [15], which is based on a simple geometry reasoning that the facet size $b$ (diameter) can be estimated by

$b=\sqrt{8 \Delta T_{\mathrm{kin}} R / G_{n}}$,

where $R$ is the radius of curvature of the melting point isotherm and $G_{n}$, local thermal gradient. However, obtaining $R$ and $G_{n}$ a priori is not possible without the detailed heat flow simulation. Therefore, in the present report, their concept for facet formation is adopted, but the interface shape and the local thermal gradients are coupled with the heat flow calculation. Apparently, the estimation of the local thermal gradient $G_{n}$ is crucial in estimating the facet size.

Once the kinetic information is known, to implement the facet calculation is straightforward. Take the growth in the $\left[\begin{array}{lll}1 & 1 & 1\end{array}\right]$ direction with $\{211\}$ facets as an example. We first align the [1 111$]$ direction with the $x$-axis. By a simple rotation transformation, the $[\overline{1} 01]$ is in the $z$ direction, while $[\overline{1} 21]$ is in the $y$-direction. Furthermore, the plane normal of the three $\{211\}$ facets can be easily obtained as well, as shown in Fig. 1b: they are $4 e_{x} / \sqrt{3}-$ $\boldsymbol{e}_{y} / \sqrt{6}+\boldsymbol{e}_{z} / \sqrt{2}, 4 \boldsymbol{e}_{x} / \sqrt{3}+\boldsymbol{e}_{y} / \sqrt{6}+\boldsymbol{e}_{z} / \sqrt{2}$, and $4 \boldsymbol{e}_{x} / \sqrt{3}-$ $\boldsymbol{e}_{y} / \sqrt{6}-\boldsymbol{e}_{z} / \sqrt{2}$, respectively, in the (1 12$)$, (1 21 ), and (211) planes. Because at the growth axis, $y=0$ and $z=0$, one can use a plane equation, i.e., $f(x, y, z)=$ $x+B y+C z+D=0$, to define each facet, where $B, C$, and $D$ are constants; $-D=x_{0}$, the intersection of the plane with the $x$-axis. The plane constants $B$ and $C$ can be easily calculated from the normal vector $(\nabla f)$; e.g., for the [ 1112$]$ plane, $B=1 /(4 \sqrt{2})$ and $C=\sqrt{3} /(4 \sqrt{2})$. During calculation, the location of $T_{\mathrm{m}}$ is found first for the rough surface, where the thermal boundary condition, Eq. (8), is used for the energy equation. Then the interface is cut by the facet plane; at the beginning one can use the melt height at $y=0$ and $z=0$ to start with. If the new interface of the cut plane is lower than the rough one, the interface shape is updated. Based the new interface shape, the temperature as well as other variables, are then updated, and the highest supercooling in the each facet can be found. If the supercooling is higher than the kinetic one, the plane is moved upward by increasing $x_{0}$. This procedure continues until all the supercooling constraints are satisfied and all the facets are tested. Because the interface is defined by the nodes as shown in Fig. 1a, the vertices are then interpolated from the node values. Both nodes and vertices are used for remeshing. This procedure is robust, and no special treatment is needed after finding the interface shape.

The above governing equations and their associated boundary conditions are solved by an efficient FVM 
scheme using multigrid acceleration [16] for the free boundary problem. Two levels of grids are used in the calculation. In the first level, there are $16 \times 21 \times 30$ (in the radial, angular, and axial directions, respectively) finite volumes in the melt and $16 \times 21 \times 15$ in the crystal, and $5 \times 21 \times 45$ in the ampoule. The second level doubles the finite volumes in each direction, i.e., $32 \times$ $42 \times 60$ cells in the melt. The calculations are performed in personal computers (PIII/1.2GHz CPU with $512 \mathrm{M}$ SRAM), and one calculation takes about $2 \mathrm{~h}$ in CPU time. For all cases, the results based on the coarse and fine grids are about the same. Detailed description of the numerical method can be found elsewhere [16].

\section{Results and discussion}

Before presenting the results for facet formation, we have performed extensive comparison with previous calculations. By using the present code, the first comparison is conducted for a simple 1D system [11] consisting of two parallel large black plates having fixed dimensionless temperatures at $\theta_{1}=1$ and $\theta_{2}=0.5$, respectively; $\theta=T / T_{1}$, where $T_{1}$ is the temperature at wall 1. Between two plates is an absorbing-emitting medium $\left(\tau_{\mathrm{L}}=a L=1 ; \epsilon_{1}=\epsilon_{2}=1\right)$ and there is no phase change. The calculated results using $P_{1}$-approximation at different conduction-to-radiation parameters $\left(N \equiv k a / 4 \sigma T_{1}^{3}\right)$ are shown in Fig. 2, where the exact solutions (solid lines) and the previous 1D $P_{1}$ calculations (thick dashed lines) are put together for comparison. As shown, they are in good agreement, except for small $N$ values $(N=0.01)$. For $N=10$, the heat conduction is dominant and the

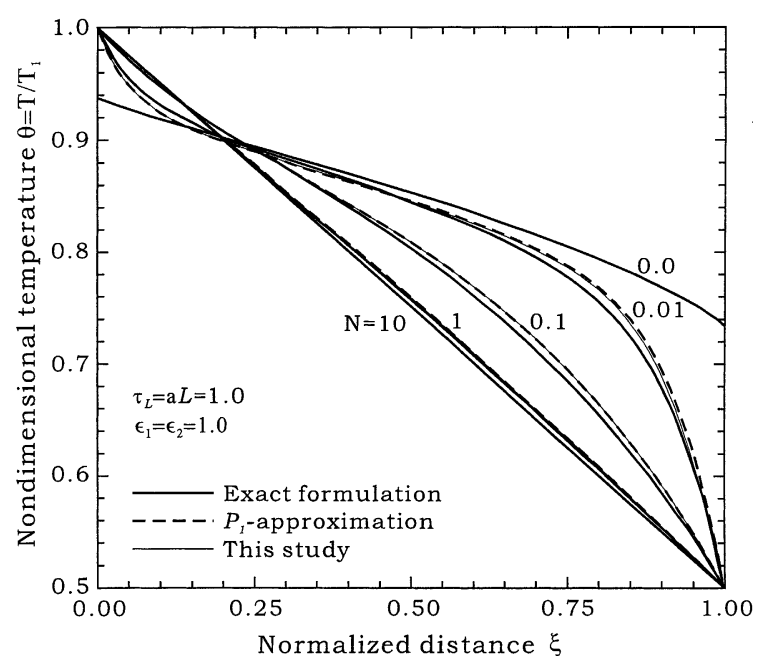

Fig. 2. Nondimensional temperature distribution for combined internal radiation and conduction across a gray slab of nondimensional optical thickness $\tau_{\mathrm{L}}=1$, between two walls with a temperature ratio of $0.5\left(\theta=T_{2} / T_{1}=0.5\right)$. thermal profile is pretty much linear. With the increasing internal radiation or decreasing $N$ value, one can see the temperature gradient increases near two walls. This is typical due to radiation cooling. Larger thermal jumps at the wall for $N=0$ indicating little emission and absorption in the medium, so that radiation takes place from surface to surface. Of course, the $P_{1}$ approximation is not suitable at this limit.

With the increase of internal radiation (smaller $N$ ), except near the boundary the temperature profile is quite linear having smaller gradients. This gives the reasoning for the Rosseland diffusion approximation using an enhanced thermal conductivity. In other words, due to internal radiation, the material tends to be more thermally conductive. However, near the walls, the Rosseland approximation starts to fail. To approximate the steep thermal gradient at the boundary, the jump boundary condition is often used. Therefore, it is clear

Table 1

Physical properties and some input parameters $[3,10]$

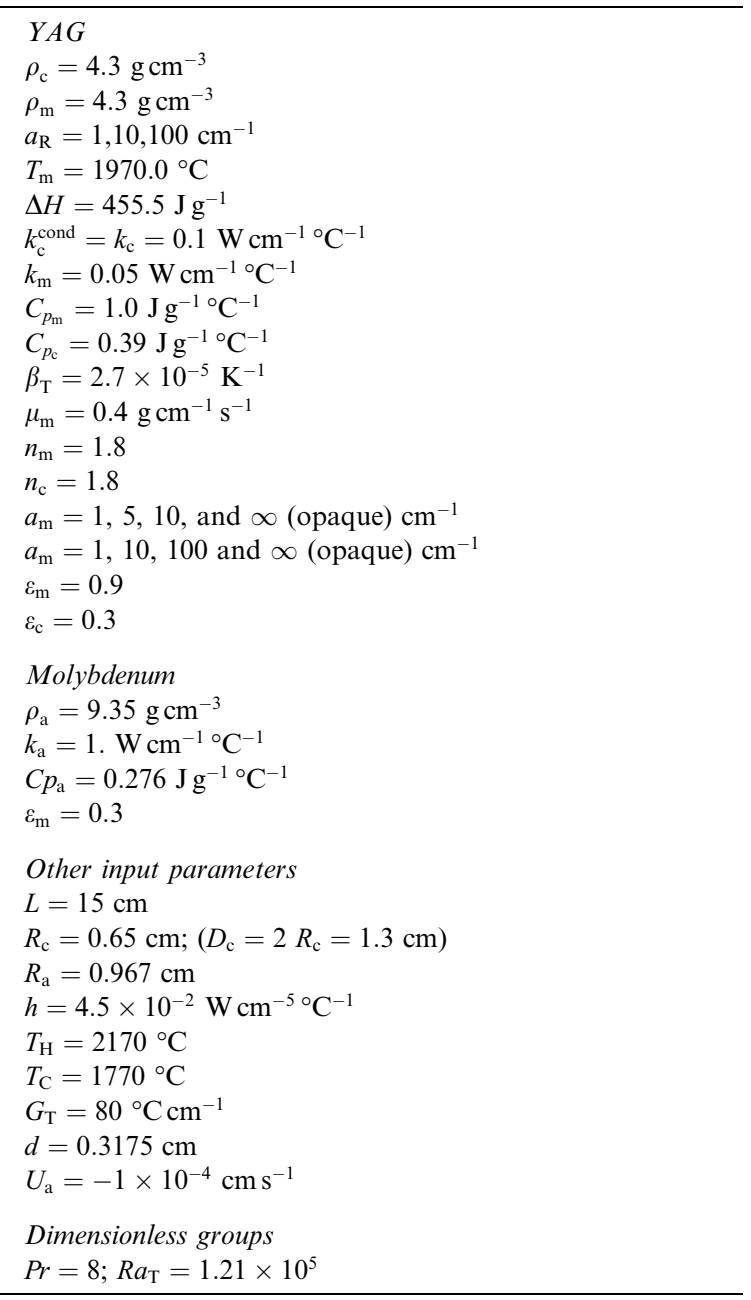


that the Rosseland approximation is not accurate near the boundary. Unfortunately, for crystal growth, the interface happens to be at the boundary, i.e., a free boundary problem. Therefore, nontrivial errors in interface calculation by the Rosseland model can be anticipated. On the other hand, the use of the jump condition at the interface complicates the numerical treatment of the Stefan boundary condition, where two temperatures are required at the interface, and the thermal gradient from heat conduction is also not realistic. Hence, the jump condition was not considered in the previous reports $[10,13,14]$. For comparison purposes, we do not introduce the slip condition as well in this report.

Although the $P_{1}$ approximation seems to be reasonable for the $1 \mathrm{D}$ problem, the applications to $2 \mathrm{D}$ or 3D problems are still uncertain, especially for crystal growth. In fact, as discussed in the previous reports [e.g., 11], the $P_{1}$ approximation performed poorly for optically thin medium, and may be substantially in error for multidimensional geometries with large aspect ratios and/or surface emission dominates over medium emission. Therefore, the second comparison is conducted for an axisymmetric configuration, which was examined carefully by Brandon and Derby [3] using a rigorous finite element approach. The data and parameters used in the simulation are summarized in Table 1 [3]. The melt here is also assumed to be opaque. Therefore, we only vary the optical absorption coefficient of crystal, i.e., $a_{\mathrm{c}}$, for comparison. Figs. 3 and 4 shows the comparison of the calculated temperature profiles at the centerline for $a_{\mathrm{c}}=10$ and $1 \mathrm{~cm}^{-1}$, respectively. As shown, for the optically thick case (or $a_{\mathrm{c}} \gg 1 \mathrm{~cm}^{-1}$ ) in

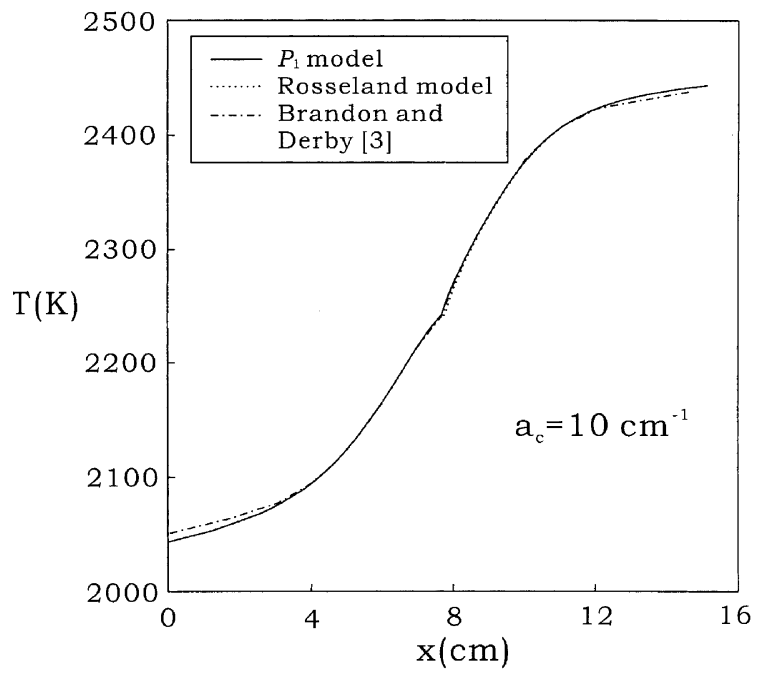

Fig. 3. Comparison of calculated axial temperature distributions (at the centerline) with the one obtained by Brandon and Derby [3] for $a_{\mathrm{c}}=10 \mathrm{~cm}^{-1}$; the melt is assumed to be opaque.

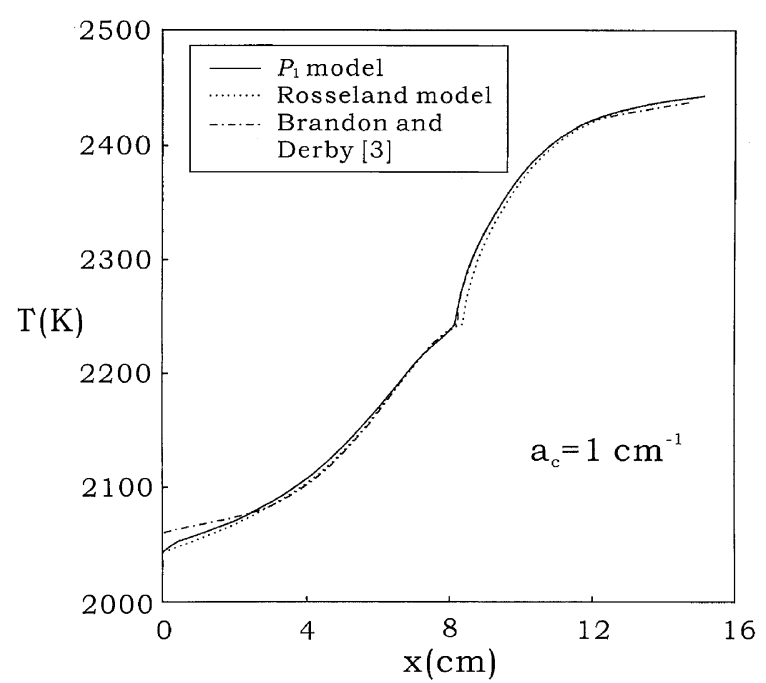

Fig. 4. Comparison of calculated axial temperature distributions (at the centerline) with the one obtained by Brandon and Derby [3] for $a_{\mathrm{c}}=1 \mathrm{~cm}^{-1}$; the melt is assumed to be opaque.

Fig. 3, the temperature profiles obtained from different models are very close. However, for $a_{\mathrm{c}}=1 \mathrm{~cm}^{-1}$ in Fig. 4 , the difference among models increases, especially near the interface. The Rosseland model over predicts the interface position, while under estimates the thermal gradient in the crystal near the interface. If we put the calculated interfaces together for comparison in Fig. 5,

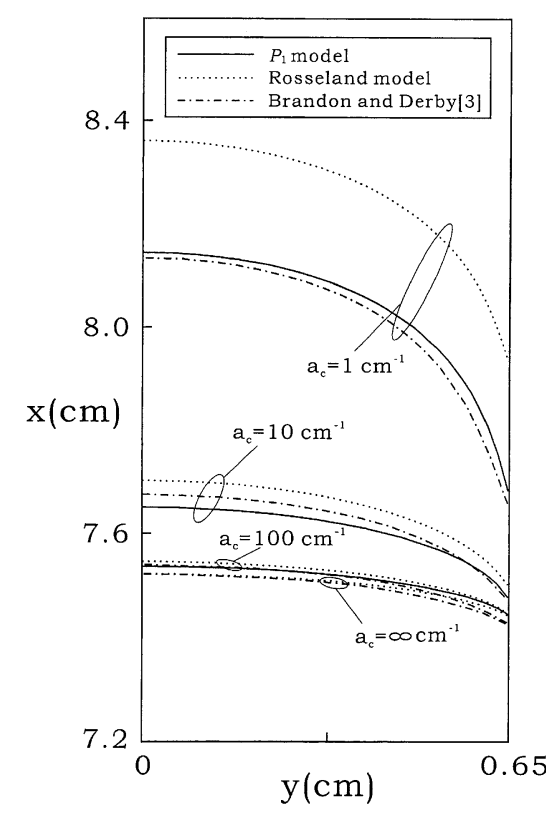

Fig. 5. Comparison of calculated interface shapes from different models for various optical absorption coefficients of the crystal; the melt is assumed to be opaque. 
the conclusion is clearer. For the optically thick situation, the agreement of the interface shapes and positions from different models is reasonably good. The discrepancy increases with the decreasing $a_{\mathrm{c}}$ value. For $a_{\mathrm{c}}=1$ $\mathrm{cm}^{-1}$, the Rosseland model over predicts the interface position, while the $P_{1}$ approximation still agrees with the rigorous model reasonably well. Nevertheless, if we just take the interface shape or convexity for comparison, surprisingly, in all cases, the calculated interface shapes are very close. In fact, this is consistent with the comparison by the Vizman et al. [13,14]. Their calculated interface shape agrees reasonably well with the one by Brandon and Derby [3]. Furthermore, as illustrated in Fig. 5, internal radiation makes the interface much more convex. As a result, as shown in Fig. 6 (only part of the domain is shown), the convection (flow velocity) increases with the interface convexity. Because YAG melt is quite viscous and the convection is not very strong, the isotherms are not distorted much in all cases. Nevertheless, the weak flow may have profound effect on dopant distribution [10]. Moreover, for oxide growth, the key issue for a highly convex interface is the supercooling and faceting that have significant effects in dopant segregation and crystal quality.

To consider the facet formation, in this study, we have chosen the growth in the [1 11 ] direction, and the typical faceting has been found at the $\{211\}$ planes [8]; the angle between [2 111 ] and [1 111 ] is about $19.47^{\circ}$. Sometimes, $\{110\}$ facets can also be found in the experiments, which occurs usually near the edge of the interface due to the larger critical angle being about $35.26^{\circ}$. For the $\{211\}$ facets, the position of faceting depends strongly on the interface shape. For a highly convex interface, they may appear near the interface center causing a core in the grown crystal. If we take faceting into account for a supercooling $\Delta T_{\text {kin }}$ of $2{ }^{\circ} \mathrm{C}$, the calculated thermal and velocity fields using the $P_{1}$ model for different $a_{\mathrm{c}}$ 's are shown in Fig. 7; two different views are shown. The $3 \mathrm{D}$ interface shapes and the temperature distributions at the interfaces are also illustrated; the darkest area in the facets has a temperature of $2{ }^{\circ} \mathrm{C}$ lower than the equilibrium melting temperature.

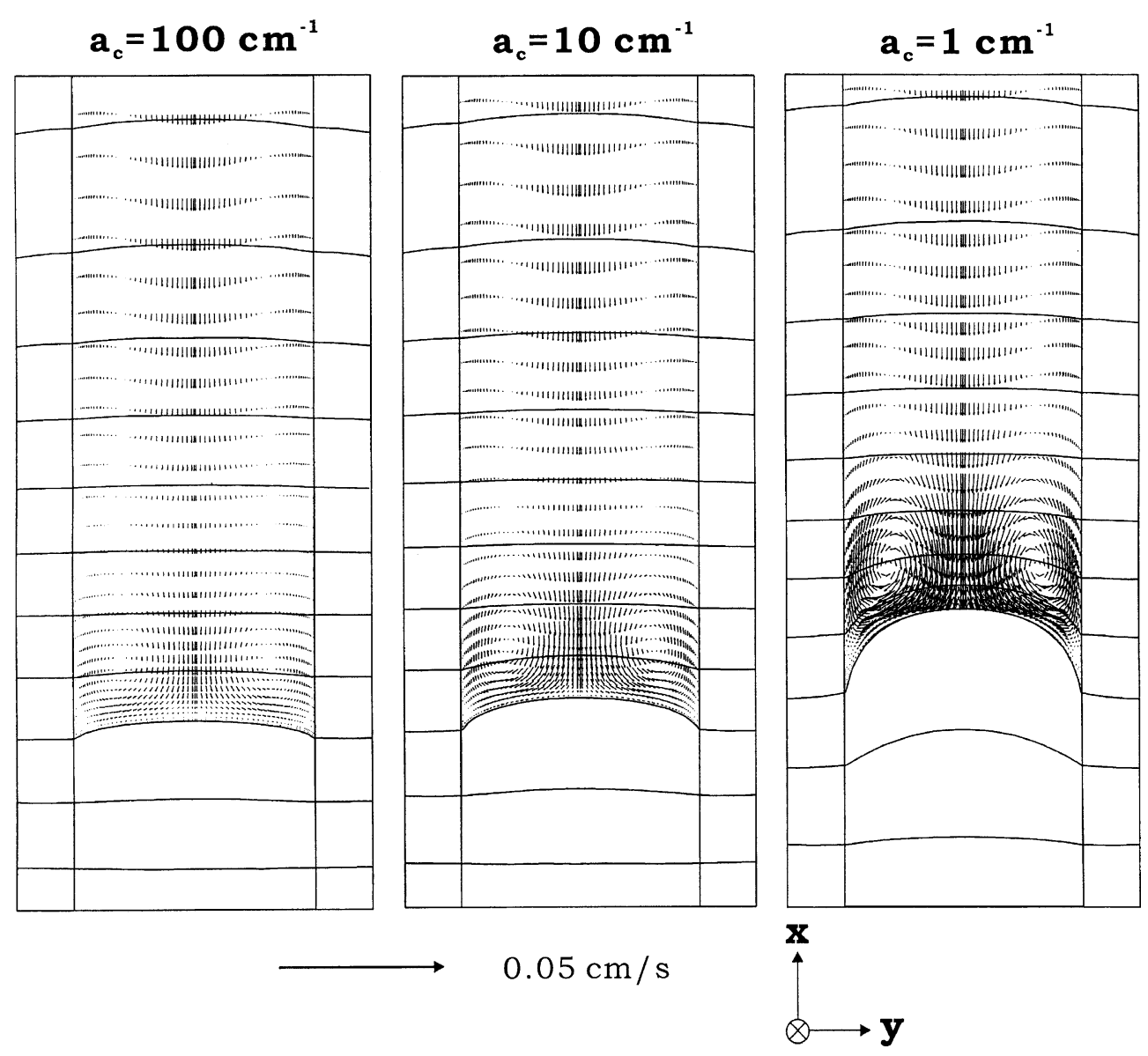

Fig. 6. Calculated thermal and flow fields for various optical absorption coefficients of the crystals by the $P_{1}$ model; the melt is assumed to be opaque. 
$a_{c}=100 \mathrm{~cm}^{-1}$
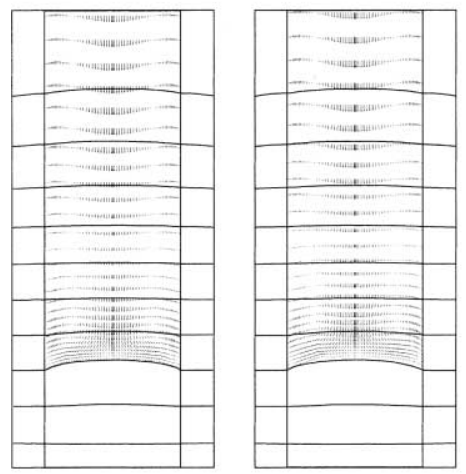

$0.05 \mathrm{~cm} / \mathrm{s}$ $a_{c}=10 \mathrm{~cm}^{-1}$
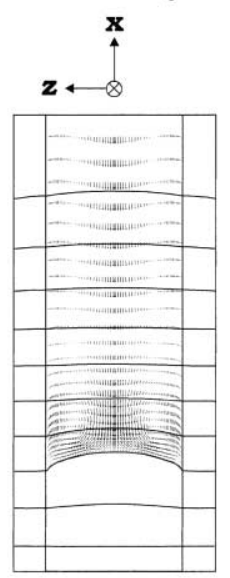

$\mathbf{a}_{\mathrm{c}}=\mathbf{1} \mathrm{cm}^{-1}$

$\stackrel{x}{x}$

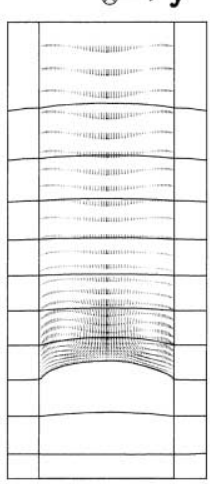

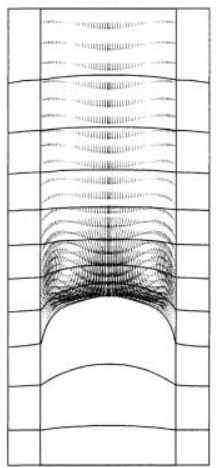
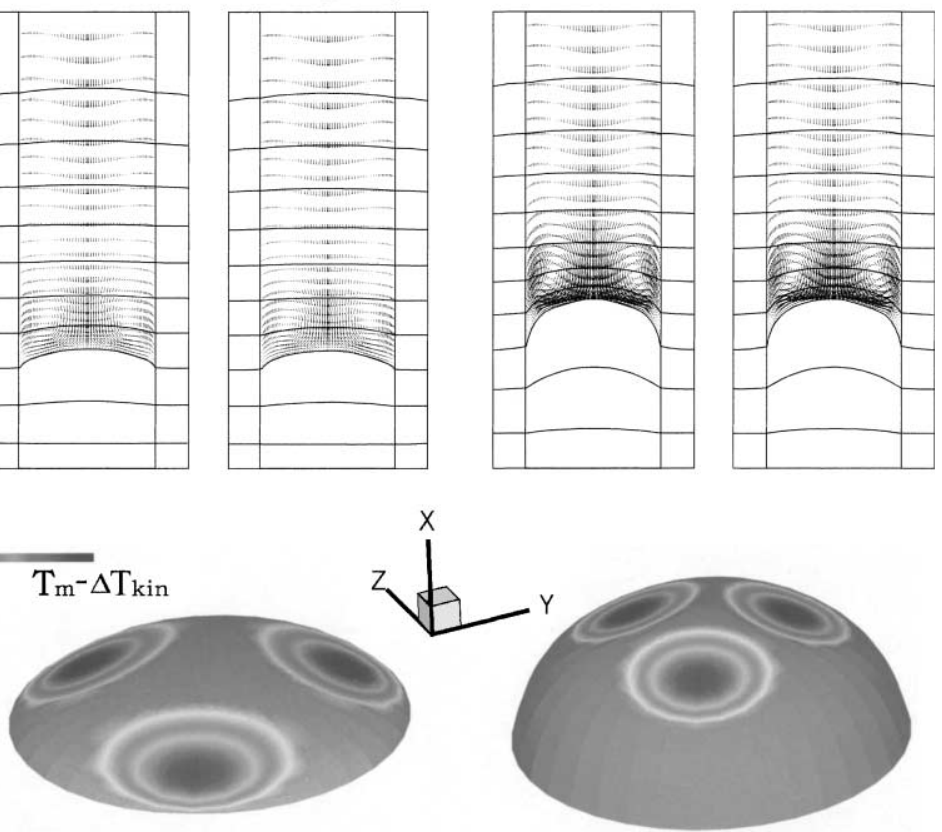

Fig. 7. Effects of absorption coefficient (internal radiation) in the crystal on the thermal and flow fields and the interface shape with $\{211\}$ facets calculated by the $P_{1}$ model: (a) $a_{\mathrm{c}}=100 \mathrm{~cm}^{-1}$; (b) $a_{\mathrm{c}}=10 \mathrm{~cm}^{-1}$; (c) $a_{\mathrm{c}}=2 \mathrm{~cm}^{-1} ; \Delta T_{\text {kin }}=2{ }^{\circ} \mathrm{C}$. The lower figures are the interfaces and the color indicates the temperature distribution; the darkest area has $2{ }^{\circ} \mathrm{C}$ supercooling.

As shown, increasing the internal radiation in the crystal pushes the interface upwards. Meanwhile, the interface becomes more convex. As a result, beside the convection in the melt is enhanced, the facet formation moves to the center part of the crystal.

The Rosselland diffusion model gives similar results, as shown in Fig. 8. However, for $a_{\mathrm{c}}=1 \mathrm{~cm}^{-1}$, i.e., a more optically thin situation, the calculated facets become much larger and three $\{211\}$ facets join at the interface center forming a singular point. Between two facets, a clear singular edge is also formed. These singular edges and point are often the position for stress concentration making the crystal much more vulnerable to thermal stress. As compared with Fig. 7, apparently the Rosseland diffusion model over predicts the facet size. As mentioned previously, due to the enhanced Rosseland thermal conductivity, the thermal gradients are underestimated near the interface in the crystal. As a result, for the same supercooling, its calculated facet size is larger. Therefore, although the calculated interface shapes by Rosseland model for the case without facets are quite close to the ones by the $P_{1}$ model, the calculated facets turn out to be erroneous. We further take the interface shapes viewed from the $x-y$ plane for comparison. As shown in Fig. 9, the difference also in- creases with the decreasing absorption coefficient of the crystal $\left(a_{\mathrm{c}}\right)$.

So far, we have assumed the melt to be opaque. This assumption is also quite typical and has been used in many calculations [3,4,10]. Also, Naso et al. [17] did some measurements showing that the melt is much less transparent as compared with the crystal. Nevertheless, many crystal growers still believe that the melt could be still transparent based on their observation. Unfortunately, an accurate measurement is still difficult and not available at this stage. Therefore, the effect of internal radiation in the melt could be interesting and may be worthwhile to study even though the experimental data is not available. Similar to the internal radiation in the crystal, clearly if the melt is transparent to radiation, the radiation from the hot zone provides a direct heating, i.e., radiational heating, to the interface. Therefore, the interface convexity should be reduced. We also consider this effect for $a_{\mathrm{c}}=1 \mathrm{~cm}^{-1}$, and the comparison of the interface shapes for different absorption coefficient of the melt $\left(a_{\mathrm{m}}\right)$ is summarized in Fig. 10a and b, for two different views, respectively. As anticipated, increasing the internal radiation in the melt reduces interface convexity as well as the position. When the melt and the crystal have the same optical thickness, the interface 


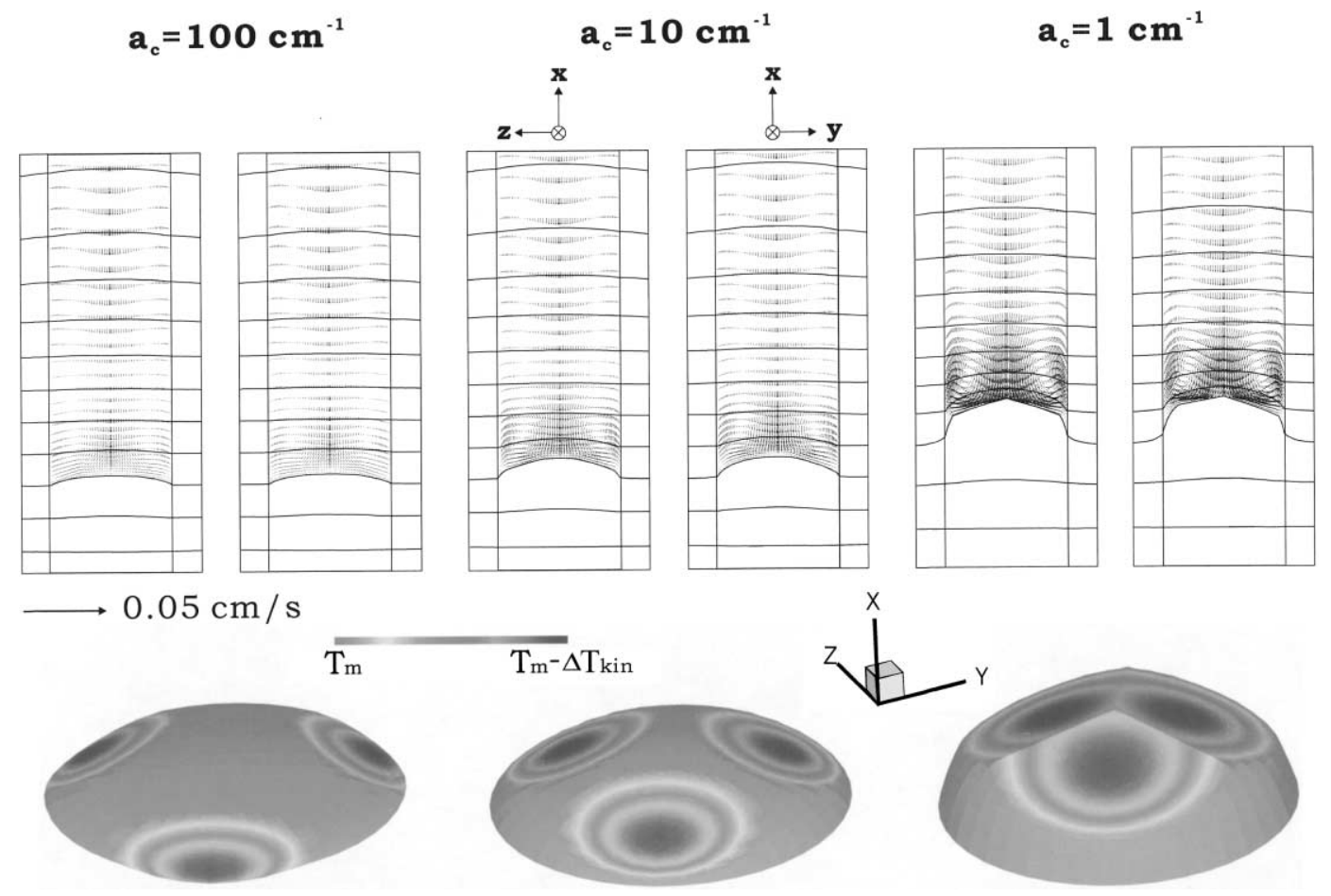

Fig. 8. Effects of absorption coefficient (internal radiation) in the crystal on the thermal and flow fields and the interface shape with $\{211\}$ facets calculated by the no-slip Rosseland diffusion model: (a) $a_{\mathrm{c}}=100 \mathrm{~cm}^{-1}$; (b) $a_{\mathrm{c}}=10 \mathrm{~cm}^{-1} ;$ (c) $a_{\mathrm{c}}=2 \mathrm{~cm}^{-1} ; \Delta T_{\mathrm{kin}}=2{ }^{\circ} \mathrm{C}$. The lower figures are the interfaces and the color indicates the temperature; the darkest area has $2{ }^{\circ} \mathrm{C}$ supercooling.

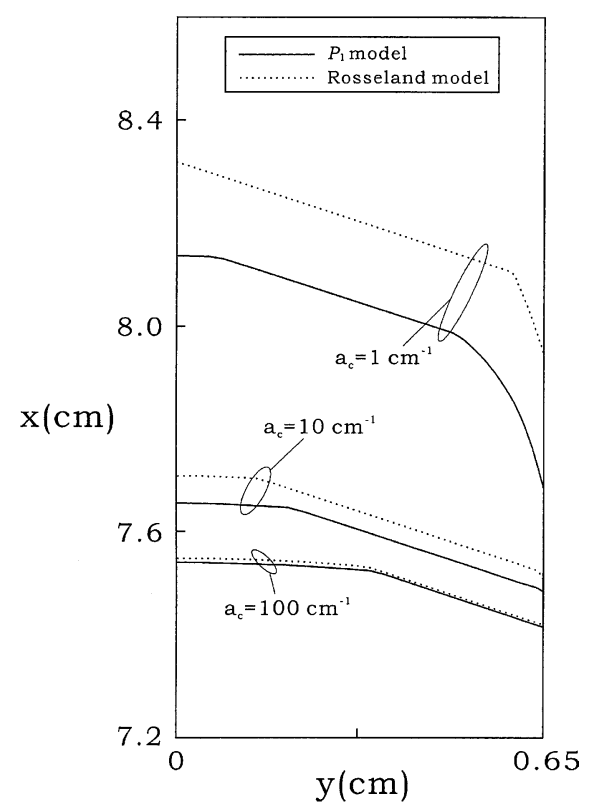

Fig. 9. Comparison of calculated interfaces by the $P_{1}$ and noslip Rosseland diffusion models for various optical absorption coefficients. shape becomes very flat; it is flatter than the case without internal radiation, even though the melt thermal conductivity is smaller. Indeed, because the radiation flux is proportional to $T^{4}$, for the same temperature difference and the optical distance, the radiational heating is larger than cooling. Similar results were also observed by Tsukada et al. [5] and Kobayashi et al. [6] for the simulation of the internal radiation in the Czochralski growth of $\mathrm{LiNbO}_{3}$ single crystals. In addition, due to the reduce of the interface convexity by radiative heating, the convection intensity is also reduced, while the facet formation moves outwards.

So far, we have illustrated that the interface convexity of YAG Bridgman growth is mainly due to the higher internal radiation in the crystal, which is particularly transparent from the thermal $1-5 \mu \mathrm{m}$ in wavelength [1]. The faceting is also calculated simultaneously based on the $P_{1}$ approximation. Still, there are other possibilities that could affect the interface convexity, such as the melt convection and the interface faceting. However, for Bridgman growth as we illustrated previously, the convection is rather weak. This is due to the thermally stable configuration of the Bridgman technique as well as the viscous melt of YAG. Therefore, this is also quite different from the cases in Czochralski growth [6], where the buoyancy flow and the forced 

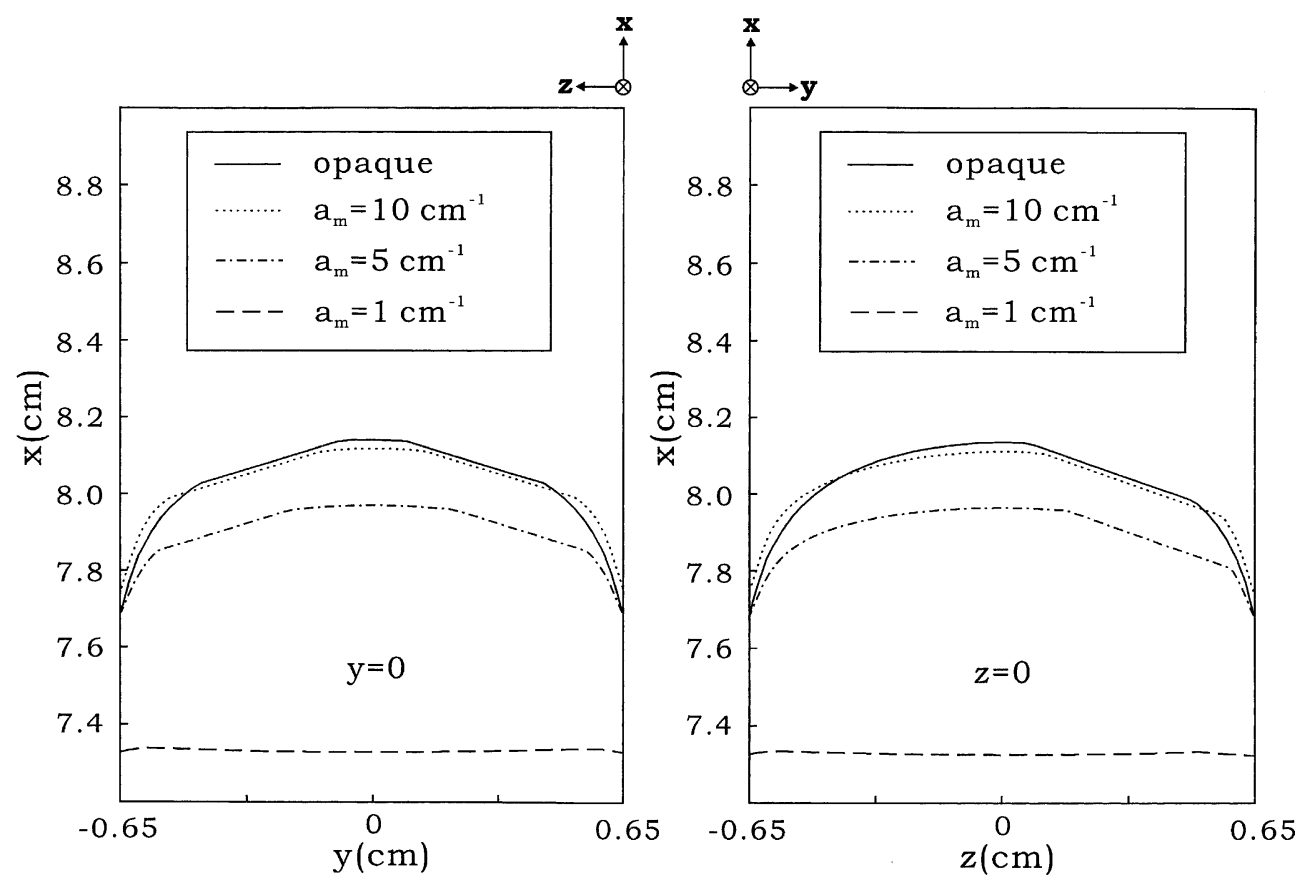

Fig. 10. Effect of absorption coefficient (internal radiation) of the melt on the interface shape for the $P_{1}$ model: (a) the interface on the $x-z$ plane; (b) on the $x-y$ plane.

convection due to crystal rotation can be the dominant factors for the interface morphology. For Bridgman growth, even with a tilted ampoule, if the tilt angle is not large, the effect of melt flow on the interface is still very limited [10]. Regarding the faceting, we have also observed that its effect on the interface convexity is not profound at all; one can also refer to the illustrations in [10] based the Rosseland diffusion model. Nevertheless, both the $P_{1}$ and the Rosseland diffusion models are suitable for optically thick materials. For a highly transparent (optically thin) material, the faceting could still be an important factor on the interface convexity. Unfortunately, this is beyond the capability of present simulation.

\section{Conclusions}

In this study, we have adopted the $P_{1}$ approximation to investigate the effects of internal radiation on the heat flow and facet formation, a formidable 3D problem. The no-slip Rosseland diffusion model is also used for comparison. In general, the calculated thermal profiles and interface shape by the $P_{1}$ approximation agree reasonable well with those by the rigorous approach for $a_{\mathrm{c}}>1 \mathrm{~cm}^{-1}$, while the Rosseland model over predicts the interface location. In addition, the thermal gradients near the interface obtained by the Rosseland model are also too small to predict reasonably the facet formation. The effect of absorption coefficient of the melt is also considered. Decreasing the melt absorbability increases radiative heating to the interface. As a result, the melt convexity as well as convection intensity decreases. The facet formation also moves outwards to the edge.

Interestingly, even though the facets break axisymmetry, the heat transfer and melt flow are pretty much symmetric. The growth at different growth directions may introduce higher asymmetry that may generate a clearer 3D flow. However, for $\{211\}$ or $\{110\}$ facets, the axisymmetry in the heat flow calculation seems to be reasonable.

\section{Acknowledgements}

The authors are grateful for the support from the National Science Council and the National Center for High Performance Computing of the Republic of China under grant no. 89-2214-E-002-040. CWL is grateful for the fruitful discussion with Prof. T. Tsukada of Tohoku University and the great hospitality of Prof. N. Imaishi for CWL's stay in the summer of 2002 sponsored by Kyushu University. Valuable comments from the reviewers are highly appreciated as well.

\section{References}

[1] B. Cockayne, M. Cheswass, D.B. Gasson, Faceting and optical perfection in Czochralski growth of garnets and ruby, J. Mater. Sci. 4 (1969) 450-456. 
[2] Ji. Kvapil, Jo. Kvapil, B. Manek, B. Berner, R. Autrata, P. Schauer, Czochralski growth of YAG: Ce in a reducing protective atmosphere, J. Crystal Growth 52 (1981) 542 545.

[3] S. Brandon, J.J. Derby, Heat transfer in vertical Bridgman growth of oxides: effects of conduction, convection and internal radiation, J. Crystal Growth 121 (1992) 473494.

[4] Q. Xiao, J.J. Derby, The role of internal radiation and melt convection in Czochralski oxide growth: deep interfaces, interface inversion, and spiraling, J. Crystal Growth 128 (1976) 188-194.

[5] T. Tsukada, K. Kakinoki, M. Hozawa, N. Imaishi, Effect of internal radiation within crystal and melt on Czochralski crystal growth of oxide, Int. J. Heat Mass Transfer 38 (1995) 2707-2714

[6] M. Kobatashi, T. Hagino, T. Tsukada, M. Hozawa, Effect of internal radiative heat transfer on interface inversion in Czochralski crystal growth of oxides, J. Crystal Growth 235 (2002) 258-270.

[7] V.S. Yuferev, M.G. Vasilev, Heat transfer in shaped thinwalled semitransparent crystals pulled from the melt, J. Crystal Growth 82 (1987) 31-38.

[8] J. Hong, N.B. Ming, Y.S. Yang, The growth of facets in Czochralski-grown $\mathrm{LiNbO}_{3}$ single crystal, Acta Physica Sinica 28 (1979) 285-292 (in Chinese).
[9] X. Xu, J. Liao, B. Shen, P. Chao, X. Chen, C. He, $\mathrm{Bi}_{12} \mathrm{SiO}_{20}$ single crystal growth by the Bridgman method, J. Crystal Growth 133 (1993) 267-272.

[10] C.W. Lan, C.Y. Tu, J. Crystal Growth 233 (2001) 523-536.

[11] M.F. Modest, Radiative Heat Transfer, McGraw-Hill, New York, 1993, p. 487.

[12] H. Matsushima, R. Viskanta, Effects of internal radiative transfer on natural convection and heat transfer in a vertical crystal growth configuration, Int. J. Heat Mass Transfer 33 (1990) 1968-2957.

[13] D. Vizman, I. Nicoara, D. Nicoara, On the factors affecting the isotherm shape during Bridgman growth of semitransparent crystals, J. Crystal Growth 169 (1996) 161-169.

[14] D. Vizman, I. Nicoara, G. Muller, Effects of temperature asymmetry and tilting in the vertical Bridgman growth of semi-transparent crystals, J. Crystal Growth 212 (2000) 334-339.

[15] V.V. Voronkov, Supercooling at the face developing on a rounded crystalization front, Krystallografiya 17 (1972) 909-917.

[16] C.W Lan, M.C. Liang, Multigrid methods for incompressible heat flow problems with an unknown interface, J. Comp. Phys. 152 (1999) 55-77.

[17] D.O. Nason, C.T. Yen, W.A. Tiller, Measurements of optical properties of some molten oxides, J. Crystal Growth 106 (1990) 221-226. 